International Journal of

Environmental Research and

Public Health

ISSN 1660-4601

www.mdpi.com/journal/ijerph

Article

\title{
Trends in Body Mass Index among Icelandic Adolescents and Young Adults from 1992 to 2007
}

Sigríður D. Eiðsdóttir ${ }^{1}$, Álfgeir L. Kristjánsson ${ }^{2}$, Inga D. Sigfúsdóttir ${ }^{2}$, Carol E. Garber ${ }^{3}$, and John P. Allegrante ${ }^{1,2,4, *}$

1 Department of Health and Behavior Studies, Teachers College, Columbia University, 525 West 120th Street, New York, NY 10027, USA; E-Mail: ste2105@columbia.edu

2 Icelandic Centre for Social Research and Analysis, School of Health and Education, Reykjavik University, Ofanleiti 2, 103 Reykjavík, Iceland; E-Mails: alfgeir@ru.is (A.L.K.); ingadora@ru.is (I.D.S.)

3 Department of Biobehavioral Sciences, Teachers College, Columbia University, 525 West 120th Street, New York, NY 10027, USA; E-Mail: ceg@2140columbia.edu

4 Department of Sociomedical Sciences, Mailman School of Public Health, Columbia University, 722 West 168th Street, New York, NY 10032, USA

* Author to whom correspondence should be addressed; E-Mail: jpa1@columbia.edu; Tel.: +1-212-678-3960; Fax: +1-212-678-8259.

Received: 23 February 2010; in revised form: 27 April 2010 / Accepted: 28 April 2010 / Published: 4 May 2010

\begin{abstract}
Trends in body mass index (BMI) among 51,889 14- to 20-year-old Icelandic adolescents and young adults were examined using data from cross-sectional population surveys conducted from 1992 to 2007. Prevalence of overweight increased for both genders in all age groups, except for 14- and 20-year-old girls. Obesity prevalence increased among boys in all age groups, except for 16-year-olds, and among 15- and 20-year-old girls. The largest increase in obesity rates among both genders was found in the oldest age group. Moreover, not only has the prevalence of obesity increased, but also the extent of obesity has grown more severe among 15- and 17-year-olds boys and among girls in the oldest age group.
\end{abstract}


Keywords: adolescents; body mass index; Iceland; obesity; overweight; prevalence

\section{Introduction}

Globally, the prevalence of overweight and obesity has increased among children, adolescents, and adults [1]. Being overweight or obese is a growing public health threat because of its significant contribution to the burden of chronic diseases, including type 2 diabetes, cardiovascular disease, hypertension, and some types of cancers [1,2]. Aside from human suffering, the economic consequences that accompany the morbidity and mortality associated with obesity are estimated to contribute up to $6 \%$ of the total healthcare costs in many of the European and other developed countries, with indirect costs in lost productivity adding equally as much $[1,3]$.

A critical developmental period for the onset of obesity is during adolescence and young adulthood [4,5]. Although the onset of obesity is less associated with morbidity in adolescence and young adulthood, it is a strong determinant of obesity and affiliated morbidity later in life, with $50 \%$ to $80 \%$ of obese adolescents growing into obese adults [6]. Adolescent onset of overweight is also associated with adult obesity-affiliated morbidity [7,8], independent of adult weight status [9]. Thus, from a public health perspective, monitoring the prevalence and trends in overweight and obesity among adolescents and young adults is of increasing importance. Furthermore, adolescents and young adults who are overweight or obese are often not only burdened by decreased capacities for physical activity [10], but they may also experience profound psychosocial complications that can affect their social interactions and school performance [11-13].

Upward trends in overweight and obesity among adolescents and young adults have been documented around the globe [14,15], and in most of the Nordic counties [16-19]; recent reports have noted stabilization in the rates of adolescent and childhood overweight and obesity from countries such as Australia [20], the United States [21], and France [22]. However, few data are available on national trends in BMI among Icelandic adolescents [19], and there are limited published studies examining changes over time in both under- and normal-weight adolescents.

In order to tailor prevention programs more effectively, a rigorous examination of the trends in adolescent onset of obesity and general trends in weight change among this age group is needed. But describing only the overweight and obese proportion of the population, or only the central part of the upper-weight distribution, provides an insufficient picture of the current situation [19,23]. In doing so, the entire distribution of body mass index (BMI) may be shifted upward, or a subgroup of the population may be seen as heavier now than before, with little change in the remainder of the population [24]. Thus, it is important to examine the shape of the whole distribution. Moreover, although prevalence rates portray the distribution of BMI, they do not provide important information about the severity of the health problem, or whether the normal, overweight, and obese are becoming heavier.

The aim of this study was to examine the trends in BMI distribution among 14- to 20-year-old Icelandic adolescents and young adults over time and within four separate weight categories during the period from 1992 to 2007. In addition, we sought to compare temporal trends in BMI in Iceland with those observed in other countries. 


\section{Methods and Procedures}

This study utilized population data from the national surveys of Icelandic adolescents, Youth in Iceland. These comprise a series of cross-sectional surveys that are conducted annually to understand trends in attitudes, values and behavior of youth along with social circumstances and background. The findings are used to enhance public understanding of youth lifestyles and to inform programs that can promote health and social well-being of Icelandic young people. The data analyzed in this study were collected in upper secondary schools at four time periods for 14- and 15-year-olds, in 1992, 1997, 2000, and 2006; and for three time periods for 16- through 20-year-olds in high-schools in 1992, 2004, and 2007. In Iceland, schooling is mandatory for youth age 6 through 15 and about 95\% of upper secondary school graduates continue their education in high-schools, which are funded by the municipalities but supervised and operated by the Ministry of Education. High school typically requires about four years to complete, usually by age 19 or 20; however, the proportion of graduates that continue in high school falls to about $85 \%$ by the second year and slightly below $70 \%$ by the 4 th and final year [25]. The distribution of the student population in Iceland is consistent with the distribution of the general population, with about $63 \%$ in the capital city, Reykjavik, and surrounding areas.

\subsection{Procedure}

The data collection in the Youth in Iceland surveys was guided by a strict methodological protocol [26]. It is carried out by the Icelandic Centre for Social Research and Analysis (ICSRA) at Reykjavik University's School of Health and Education in cooperation with the Icelandic Ministry of Education, Science, and Culture, the municipalities around the country, and the vast majority of Iceland's schools. All aspects of data collection were approved by an Icelandic central human subjects review committee, and a passive consent was required. The ICSRA administered the distribution of anonymous questionnaires to all upper secondary and high-schools in Iceland. Teachers at individual school sites assisted the students with their participation in the study and supervised the survey questionnaire returns. All students who were present in school on the survey day completed their questionnaire inside their classrooms. Upon completion, students placed and sealed the questionnaire in blank envelopes. In 1992 and 1997, the surveys were conducted by the predecessor to the ICSRA, the Institute for Educational Research, using the same protocol. Tables 1 and 2 provide information on the number of participants and response rates within each respective survey.

Table 1. Number of 14- to 15-year-olds in compulsory secondary schools who participated in the Youth in Iceland surveys, 1992, 1997*, 2000, and 2006.

\begin{tabular}{|cccc|}
\hline Year & N & \% of Population & \% Males \\
\hline 1992 & 7,018 & 85 & 50 \\
1997 & 3,913 & 45 & 52 \\
2000 & 6,346 & 82 & 49 \\
2006 & 7,430 & 82 & 50 \\
\hline
\end{tabular}

*The sample in 1997 was split in two groups (sample group A and sample group B) for methodological purposes. Questionnaires were randomly distributed to students in both groups. In the current study, we use the data from sample group A with a total of 3,913 responses. 
In total, 24,707 participants completed the four surveys in upper secondary schools.

Table 2. Number of non-compulsory high-school students who participated in the Youth in Iceland surveys, 1992*, 2004, and 2007.

\begin{tabular}{|cccc|} 
Year & $\mathrm{N}$ & $\begin{array}{c}\text { \% of eligible } \\
\text { population }\end{array}$ & \% Males \\
\hline 1992 & 4,922 & -- & 49 \\
2004 & 11,031 & 81 & 48 \\
2007 & 11,229 & 72 & 48 \\
\hline
\end{tabular}

*In 1992 the survey was based on a random sample. The eligible population is an estimate of the proportion of all dayschool students that normally attend school and is based on information from the high schools.

In total, 27,182 participants completed the three surveys in high schools.

\subsection{Filtering of Insufficient Information}

In upper secondary schools, those respondents who provided insufficient information about gender, age, height or weight, or reported their weight as less than $30 \mathrm{~kg}$ and/or above $120 \mathrm{~kg}$, were excluded from the analysis. This resulted in a total number of 22,519 participants $(91 \%$ of the participants on whom data were available across all four survey years).

Among high-school students, those who reported being below or above the 16- to 20 -years-of-age group were excluded from the analysis as were unrealistic weight and height values. Those reporting to be above $170 \mathrm{~kg}$ in weight and either under $130 \mathrm{~cm}$ or above $220 \mathrm{~cm}$ in height were therefore excluded from the analysis as well. The total number of participants in high schools numbered 21,318 (78\% of whom provide data across the three survey years).

\subsection{Measure and Classification of BMI Groups}

Self-reported height and weight were used to calculate BMI $\left(\mathrm{kg} / \mathrm{m}^{2}\right)$. For young adults, 18 years old and above, BMI was categorized according to the World Health Organization [1] classification as follows: underweight $\leq 18.5 \mathrm{~kg} / \mathrm{m}^{2}$, normal weight $=18.5-24.9 \mathrm{~kg} / \mathrm{m}^{2}$, overweight $=25-29.9 \mathrm{~kg} / \mathrm{m}^{2}$, and obese $\geq 30 \mathrm{~kg} / \mathrm{m}^{2}$. For adolescents under 18 years of age, the International Obesity Task Force (IOTF) age- and gender-specific cut-off points for underweight, overweight and obese (based on centile curves defined to pass through the BMI of $18.5,25$ and $30 \mathrm{~kg} / \mathrm{m}^{2}$ at age 18) were used to provide internationally comparable estimates of the prevalence among adolescents [27,28]. As recommended by the IOTF [28], overweight was defined as the proportion of adolescents between overweight and obese, so the overweight group does not include the obese group. Because the data were collected in March, the BMI cut-off point values for half years, 14.5, 15.5, were used for upper secondary school students. Data collection among high-school students was conducted in October, thus whole-year cut-off values were used. 


\subsection{Analysis}

Trends in average BMI between age groups and year of study are reported in Figures 1 and 2. We conducted a Chi-square test for trends in prevalence rates of underweight, normal weight, overweight and obesity within each respective age and gender group. The results are reported in Tables 3 and 4 . We also conducted a linear trend analysis using Analysis of Variance (ANOVA) for gender- and age-specific mean changes within BMI categories. These are reported in Tables 5, 6, and 7. After considering the distributional properties of the data with the normality assumptions in using ANOVA in mind, we transformed the distribution of the data with natural logarithmic calculations. As a result Skewness and Kurtosis changed from 1.26 and 2.87 to 0.64 and 1.05 respectively.

Figure 1. Mean BMI $\left(\mathrm{kg} / \mathrm{m}^{2}\right)$ of 14- and 15-year-old Icelandic adolescents in 1992, 1997, 2000, and 2006 (SD in parentheses).

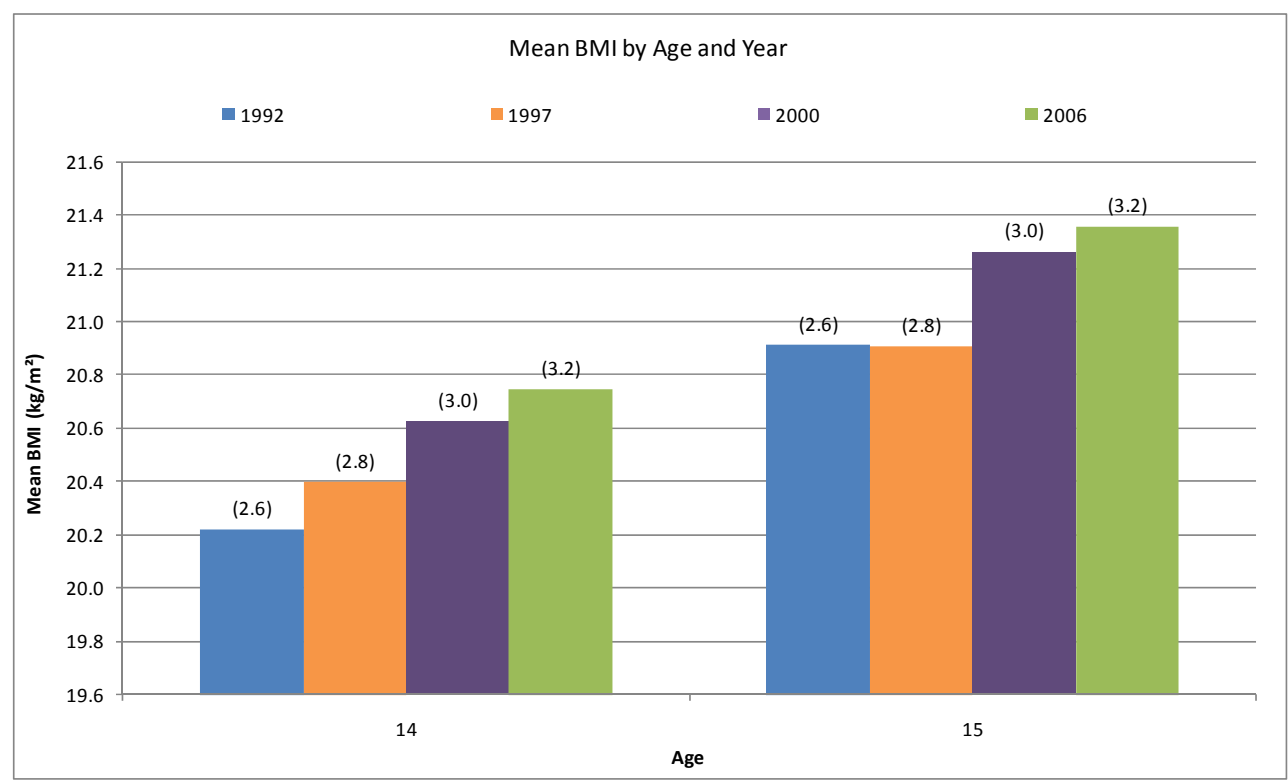

Figure 2. Mean BMI $\left(\mathrm{kg} / \mathrm{m}^{2}\right)$ of 16- to 20-year-old Icelandic adolescents in 1992, 2004 and 2007 (SD in parentheses).

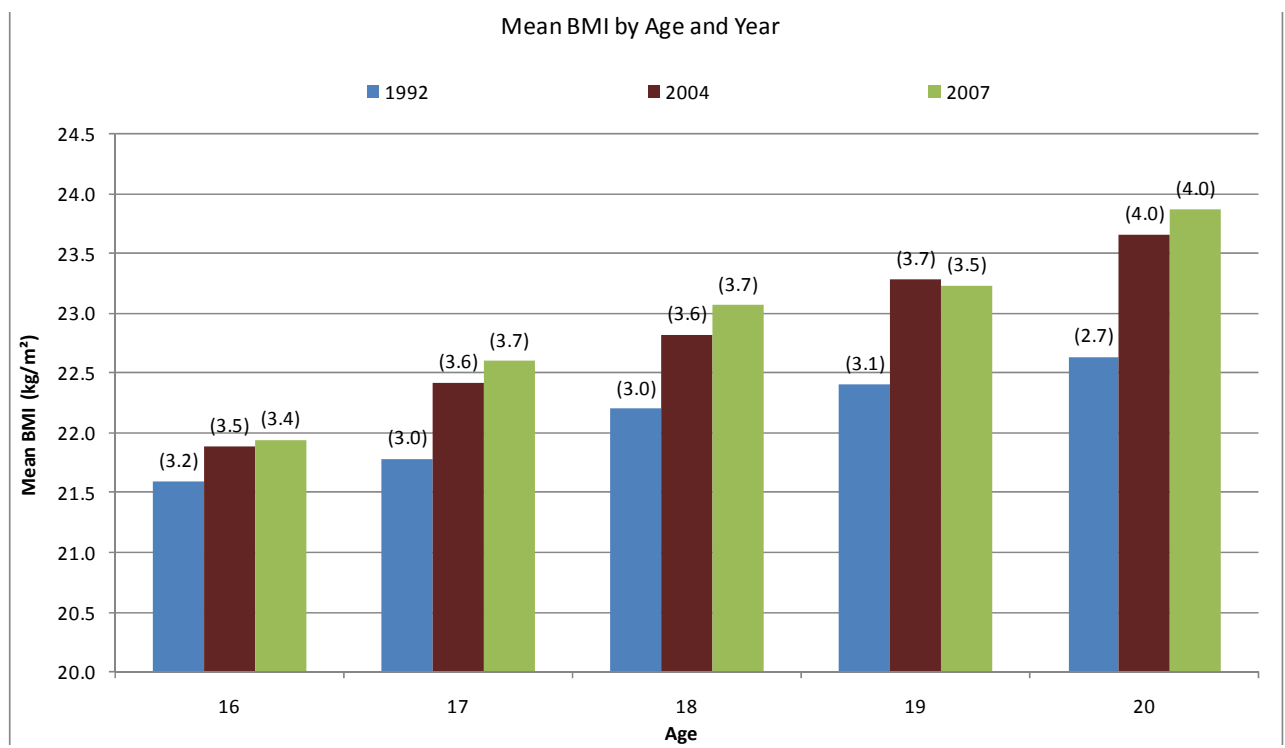


Table 3. Prevalence rates of underweight, normal weight, overweight, and obesity among 14- and 15-year-olds in 1992, 1997, 2000, and 2006.

\begin{tabular}{|c|c|c|c|c|c|}
\hline \multirow[b]{2}{*}{ BMI category } & \multirow[b]{2}{*}{ Year } & \multicolumn{2}{|c|}{ Boys } & \multicolumn{2}{|c|}{ Girls } \\
\hline & & 14 & 15 & 14 & 15 \\
\hline \multirow[t]{4}{*}{ Under } & 1992 & 3.9 & 3.1 & 10.3 & 8.9 \\
\hline & 1997 & 3.5 & 3.3 & 9.5 & 8.9 \\
\hline & 2000 & 3.5 & 2.5 & 9.1 & 9.7 \\
\hline & 2006 & 4.2 & 3.8 & 9.7 & 9.2 \\
\hline \multicolumn{2}{|l|}{ Chi-square } & $\begin{array}{c}\chi^{2}(3,5460)=1,31 \\
p=0.726\end{array}$ & $\begin{array}{c}\chi^{2}(3,5656)=4.52 \\
p=0.210\end{array}$ & $\begin{array}{c}\chi^{2}(3,5574)=1.18 \\
p=0.758\end{array}$ & $\begin{array}{c}\chi^{2}(3,5829)=0.75 \\
p=0.862\end{array}$ \\
\hline \multirow[t]{4}{*}{ Normal } & 1992 & 83.7 & 82.6 & 80.2 & 81.5 \\
\hline & 1997 & 79.7 & 82.6 & 78.8 & 80.2 \\
\hline & 2000 & 77.4 & 77.4 & 78.1 & 77.6 \\
\hline & 2006 & 74.1 & 75.9 & 77.9 & 76.5 \\
\hline \multicolumn{2}{|l|}{ Chi-square } & $\begin{array}{c}\chi^{2}(3,5460)=45.28 \\
p=0.000\end{array}$ & $\begin{array}{c}\chi^{2}(3,5656)=32.38 \\
p=0.000\end{array}$ & $\begin{array}{c}\chi^{2}(3,5574)=3.15 \\
p=0.369\end{array}$ & $\begin{array}{c}\chi^{2}(3,5829)=15.05 \\
p=0.002\end{array}$ \\
\hline \multirow[t]{4}{*}{ Over } & 1992 & 11.6 & 13.2 & 8.5 & 8.5 \\
\hline & 1997 & 15.4 & 12.0 & 10.3 & 9.4 \\
\hline & 2000 & 16.1 & 17.4 & 10.8 & 10.9 \\
\hline & 2006 & 18.1 & 16.2 & 10.4 & 11.8 \\
\hline \multicolumn{2}{|l|}{ Chi-square } & $\begin{array}{c}\chi^{2}(3,5460)=26.97 \\
p=0.000\end{array}$ & $\begin{array}{c}\chi^{2}(3,5656)=19.27 \\
p=0.000\end{array}$ & $\begin{array}{c}\chi^{2}(3,5574)=5.55 \\
p=0.136\end{array}$ & $\begin{array}{c}\chi^{2}(3,5829)=11.68 \\
p=0.009\end{array}$ \\
\hline \multirow[t]{4}{*}{ Obese } & 1992 & 0.8 & 1.0 & 1.0 & 1.1 \\
\hline & 1997 & 1.4 & 2.1 & 1.5 & 1.5 \\
\hline & 2000 & 3.0 & 2.8 & 2.0 & 1.7 \\
\hline & 2006 & 3.7 & 4.1 & 2.0 & 2.5 \\
\hline Chi-square & & $\begin{array}{c}\chi^{2}(3,5460)=35.64 \\
p=0.000\end{array}$ & $\begin{array}{c}\chi^{2}(3,5656)=32.91 \\
p=0.000\end{array}$ & $\begin{array}{c}\chi^{2}(3,5574)=6.04 \\
p=0.109\end{array}$ & $\begin{array}{c}\chi^{2}(3,5829)=9.52 \\
p=0.023\end{array}$ \\
\hline
\end{tabular}


Table 4. Prevalence rates of underweight, normal weight, overweight, and obesity among 16- to 20-year-olds in 1992, 2004 , and 2007.

\begin{tabular}{|c|c|c|c|c|c|c|c|c|c|c|c|}
\hline \multirow[b]{2}{*}{ BMI category } & \multirow[b]{2}{*}{ Year } & \multicolumn{5}{|c|}{ Boys } & \multicolumn{5}{|c|}{ Girls } \\
\hline & & 16 & 17 & 18 & 19 & 20 & 16 & 17 & 18 & 19 & 20 \\
\hline \multirow[t]{3}{*}{ Under } & 1992 & 3.2 & 3.8 & 2.5 & 1.4 & 0.4 & 6.4 & 7.9 & 10.5 & 8.6 & 6.0 \\
\hline & 2004 & 3.9 & 4.1 & 3.6 & 3.5 & 2.1 & 8.2 & 8.5 & 7.7 & 5.4 & 6.0 \\
\hline & 2007 & 3.4 & 4.1 & 4.0 & 2.8 & 2.4 & 7.7 & 8.4 & 7.4 & 6.8 & 2.9 \\
\hline Chi-square & & $\begin{array}{c}\chi^{2}(2,3079) \\
=0.70 \\
\mathrm{p}=0.704\end{array}$ & $\begin{array}{c}\chi^{2}(2,2536)= \\
0.09 \\
\mathrm{p}=0.955\end{array}$ & $\begin{array}{c}\chi^{2}(2,2154)= \\
2.00 \\
p=0.369\end{array}$ & $\begin{array}{c}\chi^{2}(2,1797)= \\
4.09 \\
\mathrm{p}=0.129\end{array}$ & $\begin{array}{c}\chi^{2}(2,833)= \\
3.55 \\
p=0.169\end{array}$ & $\begin{array}{c}\chi^{2}(2,3155)= \\
1.47 \\
\mathrm{p}=0.481\end{array}$ & $\begin{array}{c}\chi^{2}(2,2700)= \\
0.22 \\
\mathrm{p}=0.896\end{array}$ & $\begin{array}{c}\chi^{2}(2,2371)= \\
4.85 \\
\mathrm{p}=0.088\end{array}$ & $\begin{array}{c}\chi^{2}(2,2127)= \\
4.91 \\
\mathrm{p}=0.086\end{array}$ & $\begin{array}{c}\chi^{2}(2,721)= \\
2.86, \\
\mathrm{p}=0.239\end{array}$ \\
\hline \multirow[t]{3}{*}{ Normal } & 1992 & 80.4 & 81.4 & 84.7 & 81.4 & 84.2 & 82.8 & 80.2 & 77.4 & 79.8 & 78.9 \\
\hline & 2004 & 72.4 & 71.1 & 73.6 & 67.9 & 69.2 & 76.6 & 74.6 & 73.8 & 70.8 & 68.0 \\
\hline & 2007 & 71.1 & 69.9 & 70.1 & 68.2 & 61.6 & 77.6 & 74.1 & 72.4 & 73.3 & 71.2 \\
\hline Chi-square & & $\begin{array}{l}\chi^{2}(2,3079) \\
=13.03 \\
\mathrm{p}=0.001\end{array}$ & $\begin{array}{c}\chi^{2}(2,2536)= \\
23.10 \\
p=0.000\end{array}$ & $\begin{array}{c}\chi^{2}(2,2154)= \\
36.10 \\
p=0.000\end{array}$ & $\begin{array}{c}\chi^{2}(2,1797) \\
=28.12 \\
p=0.000\end{array}$ & $\begin{array}{c}\chi^{2}(2,833)= \\
34.08, \\
p=0.000\end{array}$ & $\begin{array}{c}\chi^{2}(2,3155)= \\
6.99 \\
p=0.030\end{array}$ & $\begin{array}{c}\chi^{2}(2,2770) \\
=8.12 \\
p=0.017\end{array}$ & $\begin{array}{c}\chi^{2}(2,2371) \\
=4.56 \\
p=0.102\end{array}$ & $\begin{array}{c}\chi^{2}(2,2172)= \\
12.82 \\
\mathrm{p}=0.002\end{array}$ & $\begin{array}{c}\chi^{2}(2,721)= \\
8.27 \\
\mathrm{p}=0.016\end{array}$ \\
\hline \multirow[t]{3}{*}{ Over } & 1992 & 13.5 & 13.1 & 11.3 & 15.1 & 14.6 & 6.4 & 9.0 & 9.0 & 9.0 & 12.4 \\
\hline & 2004 & 17.9 & 18.9 & 17.8 & 22.0 & 22.3 & 12.1 & 13.2 & 13.4 & 19.1 & 16.8 \\
\hline & 2007 & 20.2 & 19.7 & 19.5 & 24.0 & 27.6 & 12.0 & 13.3 & 15.9 & 15.2 & 19.5 \\
\hline Chi-square & & $\begin{array}{c}\chi^{2}(2,3079) \\
=9.17 \\
\mathrm{p}=0.010\end{array}$ & $\begin{array}{c}\chi^{2}(2,2536)= \\
10.13 \\
p=0.006\end{array}$ & $\begin{array}{c}\chi^{2}(2,2154)= \\
15.37 \\
p=0.000\end{array}$ & $\begin{array}{c}\chi^{2}(2,1797)= \\
13.16 \\
p=0.001\end{array}$ & $\begin{array}{c}\chi^{2}(2,833)= \\
13.28, \\
\mathrm{p}=0.001\end{array}$ & $\begin{array}{c}\chi^{2}(2,3155)= \\
11.32 \\
p=0.003\end{array}$ & $\begin{array}{c}\chi^{2}(2,2770)= \\
7.38 \\
p=0.025\end{array}$ & $\begin{array}{c}\chi^{2}(2,2371)= \\
14.07 \\
p=0.001\end{array}$ & $\begin{array}{c}\chi^{2}(2,2127)= \\
24.13 \\
p=0.000\end{array}$ & $\begin{array}{c}\chi^{2}(2,721)= \\
4.57 \\
\mathrm{p}=0.102\end{array}$ \\
\hline \multirow[t]{3}{*}{ Obese } & 1992 & 2.9 & 1.7 & 1.5 & 2.1 & 0.8 & 4.4 & 3.0 & 3.0 & 2.7 & 2.6 \\
\hline & 2004 & 5.8 & 5.9 & 5.0 & 6.6 & 6.5 & 3.1 & 3.6 & 5.1 & 4.7 & 9.2 \\
\hline & 2007 & 5.3 & 6.2 & 6.4 & 5.0 & 8.5 & 2.7 & 4.2 & 4.2 & 4.7 & 6.3 \\
\hline Chi-square & & $\begin{array}{c}\chi^{2}(2,3079) \\
=4.95 \\
\mathrm{p}=0.084\end{array}$ & $\begin{array}{c}\chi^{2}(2,2536)= \\
14.85 \\
p=0.001\end{array}$ & $\begin{array}{c}\chi^{2}(2,2154)= \\
16.99 \\
p=0.000\end{array}$ & $\begin{array}{c}\chi^{2}(2,1797)= \\
11.09 \\
p=0.004\end{array}$ & $\begin{array}{c}\chi^{2}(2,833)= \\
16.07, \\
p=0.000\end{array}$ & $\begin{array}{c}\chi^{2}(2,3155)= \\
3.22 \\
p=0.200\end{array}$ & $\begin{array}{c}\chi^{2}(2,2770)= \\
1.60 \\
p=0.449\end{array}$ & $\begin{array}{c}\chi^{2}(2,2371)= \\
3.47 \\
\mathrm{p}=0.176\end{array}$ & $\begin{array}{c}\chi^{2}(2,2127)= \\
3.88 \\
p=0.144\end{array}$ & $\begin{array}{c}\chi^{2}(2,721)= \\
9.99 \\
p=0.007\end{array}$ \\
\hline
\end{tabular}


Table 5. Mean BMI $\left(\mathrm{kg} / \mathrm{m}^{2}\right)$ within underweight, normal weight, overweight, and obesity among 14- and 15-year-old boys and girls in 1992, 1997, 2000, and 2006 (SD in parentheses).

\begin{tabular}{|c|c|c|c|c|c|}
\hline \multirow[b]{2}{*}{ BMI category } & \multirow[b]{2}{*}{ Year } & \multicolumn{2}{|c|}{ Boys } & \multicolumn{2}{|c|}{ Girls } \\
\hline & & 14 & 15 & 14 & 15 \\
\hline \multirow[t]{4}{*}{ Under } & 1992 & $16.0(0.7)$ & $16.5(0.6)$ & $16.3(0.8)$ & $16.8(0.7)$ \\
\hline & 1997 & $15.7(0.8)$ & $16.4(0.9)$ & $16.3(0.6)$ & $16.7(0.9)$ \\
\hline & 2000 & $15.9(0.7)$ & $16.3(0.9)$ & $16.4(0.8)$ & $16.7(0.8)$ \\
\hline & 2006 & $15.8(0.6)$ & $16.2(0.9)$ & $16.2(0.8)$ & $16.6(0.9)$ \\
\hline \multirow{2}{*}{\multicolumn{2}{|c|}{ Linear trend }} & $F(1,3)=2.32$ & $F(1,3)=2.52$ & $F(1,3)=0.29$ & $F(1,3)=4.13$ \\
\hline & & $p=0.129$ & $\mathrm{p}=0.114$ & $\mathrm{p}=.594$ & $\mathrm{p}=0.043$ \\
\hline \multirow[t]{4}{*}{ Normal } & 1992 & $19.8(1.5)$ & $20.4(1.5)$ & $20.0(1.6)$ & $20.5(1.6)$ \\
\hline & 1997 & $19.7(1.5)$ & $20.4(1.5)$ & $19.9(1.6)$ & $20.5(1.7)$ \\
\hline & 2000 & $20.0(1.6)$ & $20.6(1.6)$ & $19.9(1.7)$ & $20.5(1.7)$ \\
\hline & 2006 & $19.9(1.6)$ & $20.6(1.6)$ & $20.1(1.7)$ & $20.5(1.7)$ \\
\hline \multirow{2}{*}{\multicolumn{2}{|c|}{ Linear trend }} & $F(1,3)=6.49$ & $F(1,3)=12.78$ & $F(1,3)=2.63$ & $F(1,3)=0.18$ \\
\hline & & $\mathrm{p}=0.011$ & $\mathrm{p}=0.000$ & $\mathrm{p}=0.105$ & $\mathrm{p}=0.669$ \\
\hline \multirow[t]{4}{*}{ Over } & 1992 & $24.7(1.2)$ & $25.3(1.3)$ & $25.4(1.3)$ & $25.9(1.3)$ \\
\hline & 1997 & $24.6(1.4)$ & $25.0(1.2)$ & $25.4(1.4)$ & $26.0(1.4)$ \\
\hline & 2000 & $24.7(1.4)$ & $25.4(1.4)$ & $25.4(1.3)$ & $25.9(1.3)$ \\
\hline & 2006 & $24.8(1.3)$ & $25.4(1.4)$ & $25.3(1.4)$ & $260.0(1.3)$ \\
\hline \multirow{2}{*}{\multicolumn{2}{|c|}{ Linear trend }} & $F(1,3)=1.14$ & $F(1,3)=2.72$ & $F(1.3)=0.21$ & $F(1,3)=0.45$ \\
\hline & & $\mathrm{p}=0.287$ & $\mathrm{p}=0.100$ & $\mathrm{p}=0.644$ & $\mathrm{p}=0.505$ \\
\hline \multirow[t]{4}{*}{ Obese } & 1992 & $30.6(1.4)$ & $30.0(1.2)$ & $31.4(1.6)$ & $31.2(1.4)$ \\
\hline & 1997 & $30.9(2.1)$ & $30.3(1.5)$ & $30.7(1.3)$ & $30.7(1.0)$ \\
\hline & 2000 & $30.6(1.9)$ & $30.8(1.7)$ & $30.7(1.5)$ & $31.2(1.5)$ \\
\hline & 2006 & $30.8(2.1)$ & $30.9(1.8)$ & $31.3(1.5)$ & $31.2(1.5)$ \\
\hline \multicolumn{2}{|l|}{ Linear trend } & $\begin{array}{c}F(1,3)=0.17 \\
\mathrm{p}=0.684\end{array}$ & $\begin{array}{c}F(1,3)=5.20 \\
\mathrm{p}=0.024\end{array}$ & $\begin{array}{c}F(1.3)=0.07 \\
\mathrm{p}=0.793\end{array}$ & $\begin{array}{c}F(1.3)=0.17 \\
p=0.680\end{array}$ \\
\hline
\end{tabular}

Table 6. Mean BMI $\left(\mathrm{kg} / \mathrm{m}^{2}\right)$ within underweight, normal weight, overweight, and obesity among 16- to 20-year-old boys in 1992, 2004, and 2007 (SD in parentheses).

\begin{tabular}{|c|c|c|c|c|c|c|}
\hline Boys & \multirow{2}{*}{ Year } & \multicolumn{5}{|c|}{ Age } \\
\hline BMI category & & 16 & 17 & 18 & 19 & 20 \\
\hline \multirow[t]{3}{*}{ Under } & 1992 & $17.0(0.7)$ & $17.2(0.8)$ & $17.5(0.8)$ & $17.7(0.6)$ & N/A \\
\hline & 2004 & $16.3(1.1)$ & $17.1(1.1)$ & $17.6(0.9)$ & $17.2(1.5)$ & $17.6(0.7)$ \\
\hline & 2007 & $16.3(1.3)$ & $16.6(1.5)$ & $17.5(1.0)$ & $17.3(1.4)$ & $16.6(0.9)$ \\
\hline & & $F(1,2)=3.59$ & $F(1,2)=2.36$ & $F(1.2)=0.00$ & $F(1,2)=0.57$ & $F(1,2)=1.92$ \\
\hline Linear trend & & $\mathrm{p}=0.061$ & $\mathrm{p}=0.128$ & $\mathrm{p}=0.948$ & $\mathrm{p}=0.456$ & $\mathrm{p}=0.193$ \\
\hline \multirow[t]{3}{*}{ Normal } & 1992 & $21.1(1.5)$ & $21.4(1.6)$ & $22.0(1.5)$ & $22.0(1.5)$ & $22.5(1.5)$ \\
\hline & 2004 & 20.9 (1.6) & $21.5(1.6)$ & $22.0(1.7)$ & $22.2(1.6)$ & $22.1(1.7)$ \\
\hline & 2007 & $21.0(1.5)$ & $21.6(1.6)$ & $22.0(1.7)$ & $22.3(1.6)$ & $22.3(1.7)$ \\
\hline & & $F(1,2)=0.84$ & $F(1,2)=3.75$ & $F(1,2)=0.04$ & $F(1,2)=4.33$ & $F(1,2)=4.38$ \\
\hline Linear trend & & $\mathrm{p}=0.356$ & $\mathrm{p}=0.053$ & $\mathrm{p}=0.842$ & $\mathrm{p}=0.038$ & $\mathrm{p}=0.037$ \\
\hline
\end{tabular}


Table 6. Cont.

\begin{tabular}{|lcccccc|}
\hline Over & 1992 & $25.2(1.3)$ & $26.1(1.4)$ & $27.0(1.4)$ & $26.6(1.3)$ & $26.4(1.2)$ \\
& 2004 & $25.8(1.3)$ & $26.3(1.4)$ & $26.8(1.4)$ & $26.7(1.3)$ & $26.9(1.2)$ \\
& 2007 & $25.6(1.2)$ & $26.3(1.3)$ & $26.9(1.4)$ & $26.9(1.4)$ & $27.0(1.3)$ \\
\hline \multirow{2}{*}{ Linear trend } & $F(1,2)=4.79$ & $F(1,2)=1.16$ & $F(1,2)=0.25$ & $F(1,2)=2.12$ & $F(1,2)=5.45$ \\
\hline Obese & & $\mathrm{p}=0.029$ & $\mathrm{p}=0.281$ & $\mathrm{p}=0.619$ & $\mathrm{p}=0.146$ & $\mathrm{p}=0.021$ \\
& 1992 & $31.0(2.1)$ & $31.3(1.3)$ & $32.1(2.4)$ & $32.1(2.4)$ & $31.0(0.7)$ \\
& 2004 & $32.3(2.9)$ & $32.1(2.4)$ & $33.2(2.7)$ & $33.3(2.1)$ & $32.8(2.0)$ \\
& 2007 & $32.1(3.1)$ & $33.3(2.8)$ & $33.2(2.5)$ & $32.6(2.7)$ & $32.7(2.2)$ \\
\hline & & $F(1,2)=1.29$ & $F(1,2)=6.68$ & $F(1,2)=1.03$ & $F(1,2)=0.27$ & $F(1,2)=1.00$ \\
Linear trend & & $\mathrm{p}=0.258$ & $\mathrm{p}=0.011$ & $\mathrm{p}=0.312$ & $\mathrm{p}=0.608$ & $\mathrm{p}=0.323$ \\
\hline
\end{tabular}

$\mathrm{N} / \mathrm{A}=$ there were no 20-year-old boys underweight in 1992.

Table 7. Mean BMI $\left(\mathrm{kg} / \mathrm{m}^{2}\right)$ within underweight, normal weight, overweight, and obesity among 16- to 20-year-old girls in 1992, 2004, and 2007.

\begin{tabular}{|c|c|c|c|c|c|c|}
\hline \multirow{2}{*}{$\begin{array}{c}\text { Girls } \\
\text { BMI category }\end{array}$} & \multirow{2}{*}{ Year } & \multicolumn{5}{|c|}{ Age } \\
\hline & & 16 & 17 & 18 & 19 & 20 \\
\hline \multirow[t]{3}{*}{ Under } & 1992 & $17.1(0.7)$ & $17.5(0.6)$ & $17.7(0.7)$ & $17.7(0.6)$ & $17.7(0.8)$ \\
\hline & 2004 & $17.0(0.8)$ & $17.4(0.7)$ & $17.4(1.1)$ & $17.6(0.8)$ & $18.1(0.3)$ \\
\hline & 2007 & $16.9(1.0)$ & $17.3(0.8)$ & $17.6(0.8)$ & $17.5(1.1)$ & $17.0(1.2)$ \\
\hline \multirow{2}{*}{\multicolumn{2}{|c|}{ Linear trend }} & $F(1,2)=1,59$ & $F(1,2)=1.55$ & $F(1,2)=1.32$ & $F(1,2)=1.62$ & $F(1,2)=0.01$ \\
\hline & & $\mathrm{p}=0.208$ & $\mathrm{p}=0.215$ & $\mathrm{p}=0.252$ & $\mathrm{p}=0.205$ & $\mathrm{p}=0.917$ \\
\hline \multirow[t]{3}{*}{ Normal } & 1992 & 20.7 (1.7) & $21.0(1.6)$ & $21.4(1.6)$ & $21.5(1.6)$ & $21.5(1.7)$ \\
\hline & 2004 & $20.8(1.7)$ & $21.2(1.7)$ & $21.5(1.7)$ & $21.6(1.7)$ & $21.7(1.8)$ \\
\hline & 2007 & $20.9(1.7)$ & $21.4(1.7)$ & $21.6(1.7)$ & $21.7(1.6)$ & $21.6(1.7)$ \\
\hline \multirow{2}{*}{\multicolumn{2}{|c|}{ Linear trend }} & $F(1,2)=2.27$ & $F(1,2)=10.52$ & $F(1,2)=5.58$ & $F(1,2)=5.90$ & $F(1,2)=0.39$ \\
\hline & & $\mathrm{p}=0.132$ & $\mathrm{p}=0.001$ & $\mathrm{p}=0.018$ & $\mathrm{p}=0.015$ & $\mathrm{p}=0.550$ \\
\hline \multirow[t]{3}{*}{ Over } & 1992 & $26.1(1.4)$ & $26.0(1.2)$ & $26.6(1.3)$ & $27.0(1.2)$ & $26.7(1.4)$ \\
\hline & 2004 & $26.3(1.3)$ & $26.6(1.4)$ & $26.7(1.2)$ & $26.9(1.4)$ & $27.0(1.3)$ \\
\hline & 2007 & $26.4(1.4)$ & $26.5(1.4)$ & $26.8(1.3)$ & $26.9(1.4)$ & $26.7(1.4)$ \\
\hline \multirow{2}{*}{\multicolumn{2}{|c|}{ Linear trend }} & $F(1,2)=1.82$ & $F(1,2)=6.11$ & $F(1,2)=0.85$ & $F(1,2)=0.13$ & $F(1,2)=0.12$ \\
\hline & & $\mathrm{p}=0.178$ & $\mathrm{p}=0.014$ & $\mathrm{p}=0.356$ & $\mathrm{p}=0.718$ & $\mathrm{p}=0.730$ \\
\hline \multirow[t]{3}{*}{ Obese } & 1992 & 33.9 (2.9) & 33.7 (2.7) & $34.2(2.5)$ & $34.9(2.3)$ & $31.3(1.2)$ \\
\hline & 2004 & $32.6(2.8)$ & $33.6(3.1)$ & $32.7(2.3)$ & $33.5(2.6)$ & 33.9 (2.9) \\
\hline & 2007 & $32.8(2.9)$ & $32.8(2.7)$ & $33.3(2.7)$ & $33.1(2.7)$ & $34.3(3.4)$ \\
\hline & & $F(1,2)=2.16$ & $F(1,2)=0.99$ & $F(1,2)=1.83$ & $F(1,2)=4.28$ & $F(1,2)=5.44$ \\
\hline Linear trend & & $\mathrm{p}=0.145$ & $\mathrm{p}=0.322$ & $\mathrm{p}=0.180$ & $\mathrm{p}=0.042$ & $\mathrm{p}=0.025$ \\
\hline
\end{tabular}

\section{Results}

Mean BMI increased consecutively over the study period for both genders across all age groups. As shown in Figures 1 and 2, the increase was most profound among 17- to 20-year-olds. Mean BMI for both 14 - and 15 -year-olds increased by $0.5 \mathrm{~kg} / \mathrm{m}^{2}$ from $1992-2006$ as shown in Figure 1 . The same trend was observed among16- to 20-year-olds. From 1992 to 2007, average BMI increased between 
0.4-1.2 kg/m² respectively, for the 16-, 17-, 18-, 19- and 20-year-olds. The biggest increase was found in the oldest age group.

\subsection{Under- and Normal Weight}

As the first columns in Tables 3 and 4 reveal, there were no statistically significant changes in the proportion of underweight in any age group for either gender, while a significant reduction in rates of normal weight was detected across all age groups for both genders, except from the 14- and 18-year-old girls. However, the changes in rates were slightly u-shaped for the 19-year-old boys, and 16-, 19-, and 20-year-old girls. The biggest decline in normal weight was found among 20 -year-old boys, a reduction from $84.2 \%$ to $61.1 \%$, and the most notable shift in girls was also among the 20 -year-olds, a decline from $78.9 \%$ in 1992 to a $68.0 \%$ in 2004 , and up to $71.2 \%$ in 2007 .

\subsection{Overweight and Obesity}

As shown in the third columns in Tables 3 and 4, there was a statistically significant increase in overweight during the study period among boys in all age groups and among girls in all age groups, except for the 14- and 20-year-olds. The greatest increase was found among boys in the oldest age group, an increase from $14.6 \%$ to $27.6 \%$. Further, obesity rates increased significantly among boys in all age groups, except for 16-year-olds, and among 15 and 20 -year-old girls. The biggest change in obesity rates among both genders was found in the oldest group; from $0.8 \%$ to $8.5 \%$ among the boys and from $2.6 \%$ in 1992 up to $9.2 \%$ in 2004 and down to $6.3 \%$ in 2007 among the girls.

\subsection{Within Each Weight Category}

As the first columns in Tables 5, 6, and 7 show, there were no statistically significant trends in the underweight category apart from the 15-year-old girls, where the average BMI decreased slightly, or by $0.2 \mathrm{~kg} / \mathrm{m}^{2}$ over the study period. In the normal-weight category, small but statistically significant increases of $0.1,0.2$, and $0.3 \mathrm{~kg} / \mathrm{m}^{2}$, were observed among 14-, 15-, and 19-year-old boys, respectively, while a $0.2 \mathrm{~kg} / \mathrm{m}^{2}$ u-shaped decrease, from 22.5 down to 22.1 and up to $22.3 \mathrm{~kg} / \mathrm{m}^{2}$ in 2007 , was observed among the 20 -year-old boys. Similarly, among girls, small but statistically significant increases of $0.4,0.2$, and $0.2 \mathrm{~kg} / \mathrm{m}^{2}$ were found among the 17-, 18-, and 19-year-olds, respectively. In the overweight category a significant increase in average BMI were observed among 16- and 20-year-old boys and 17-year-old girls, in whom BMI increased by $0.4,0.6$, and $0.5 \mathrm{~kg} / \mathrm{m}^{2}$, respectively. In the obese category, there was an increase in average BMI for boys in all age groups but only statistically significantly so among the 15- and 17-year-olds, where the average BMI increased by 0.9 and $2 \mathrm{~kg} / \mathrm{m}^{2}$, respectively. In contrast, among girls in the obese category there was a slight decrease in average BMI across all age groups, but only significantly so among the 19-year-olds, by $1.8 \mathrm{~kg} / \mathrm{m}^{2}$, with the exception of 20 -year-old girls, who experienced a mean increase of $3 \mathrm{~kg} / \mathrm{m}^{2}$.

\section{Discussion}

This is the first study to present national trend and prevalence data for underweight, normal weight, overweight, and obese Icelandic adolescents and young adults. Our results show a clear shift away 
from normal weight status. The findings reveal an increased prevalence of overweight between 1992 and 2006/2007 for both genders in all age groups, except for 14- and 20-year-old girls. Obesity prevalence also increased among boys in all age groups, except for 16-year-olds, and among 15- and 20-year-old girls. Moreover, not only did the prevalence of obesity increase, but the extent of obesity grew more severe among 15- and 17-year-olds boys and among girls in the oldest age group. These results are worrisome because health-care costs rise more sharply with higher BMI in the obese than the normal-weight population [29], and recent projections suggest a reduction in life expectancy will occur as a result of this [30].

Our results are also particularly striking in light of several recent reports that have shown a plateau in the prevalence of overweight and obesity among adolescents [20-22]. However, a parallel trend has been reported among the Icelandic adult population from 1990 to 2007, where the rate of obesity in males rose from $7.2 \%$ to $18.9 \%$ and from $9.5 \%$ to $21.3 \%$ in females, with $66.6 \%$ of males and $53.3 \%$ of females being overweight and obese in 2007 [31]. Rankings among 17 European countries showed overweight and obesity levels above $20 \%$ in seven countries among adolescents aged 13 to 17 years [32]. England reached the highest prevalence point at $25 \%$, followed by, Italy, Cyprus, Ireland, Greece, Bulgaria, and Spain. Denmark and Hungary reported overweight and obesity levels of 15\% to $20 \%$, while Poland and Finland reported rates of 10 to $15 \%$ among this age group [32]. In comparison, our findings display an $18.4 \%$ prevalence of overweight and obesity among 14- to 17-year-olds in 2006/2007; however, as the survey years differ, the comparison requires some caution when interpreting [32].

Our results also display an unfavorable pattern of overweight and obesity with increased age. Among adolescents from 14- to 20-years-old, the prevalence of being overweight and obese increased from $21.8 \%$ to $36.1 \%$ among boys and from $12.4 \%$ to $25.8 \%$ among girls, with more than one-third of 20 -year-old boys and over one-fourth of 20-year-old girls being overweight or obese in 2007. Our findings point to the threat to health of the population, whose impact is likely to grow even more serious because parental obesity - a known risk factor for childhood obesity [33] — is increasing concurrently and obesity in children and adolescents is linked to poor health outcomes in adulthood [7-9]. Additionally, the prevalence of rising obesity in older-adolescent and young-adult age groups will likely result in a higher number of pregnant women being overweight or obese. Obese mothers are much more likely to have children with high birth weights [34], which, in turn, can set the stage for obesity later in life; as shown in the cohorts born in Iceland in 1988 and 1994, children who weighed above the 85 th percentile at birth were more likely than others to be overweight at the ages of 6,9 , and 15 years [35].

According to an Icelandic dietary consumption survey, young people — especially young men — dine less at home with their families than in previous years, and they also have the highest rates of fastfood, prepared-meal, and sugar consumption in the population [36]. Physical inactivity is an important determinant of overweight throughout the western world [37,38], even after controlling for dietary patterns [37], and it may play an important role in the trends observed in the BMI distribution in this study. In a recent study of the same population over the same time period, a $6 \%$ increase in the rate of vigorous physical activity was observed, with a $15 \%$ increase in active sports club participation from 1992 to 2006 among 14- to 15-year olds [39]. However, despite these levels of reported activity, over half of the adolescents did not achieve the recommended levels of participation in physical activities. 
Furthermore, there was also an overall increase in the proportion of inactive adolescents over time, with girls consistently reporting higher levels of physical inactivity compared with boys, even though the net increase in physical inactivity was higher for boys over this time period [39]. This trend is consistent with the clearly increasing prevalence of overweight and obese boys found in this study. Although the number of health clubs, recreational facilities, and organized leisure-time physical activities have all increased over time, there is a paradoxical decrease in the levels of daily physical activity experienced in daily lives.

Taken together, our findings suggest that Iceland may be facing an increasing burden due to chronic diseases associated with overweight and obesity. As the severity of overweight and obesity increases, so do the risks of adult health problems [40-42]. Childhood obesity is associated with an increase in metabolic abnormalities, including insulin resistance, type 2 diabetes mellitus, dyslipidemia, hypertension and polycystic ovary syndrome that begin in childhood and continue into adulthood [43]. In adults, the health consequences of obesity can be particularly devastating. For example, a one-unit increase in BMI is associated with an increased risk of heart failure at the rate of 5\% in adult men and $7 \%$ in adult women [44], and is associated with a $6 \%$ increase in the relative risks for ischemic and hemorrhagic stroke [45]. Adverse health consequences occur not only among those who are in overweight and obese categories; disease risk starts to increase even for those at the upper end of the normal range (BMI of between 22 and 24.9) [41,44]. For example, it is recommended that adults maintain a BMI between 18.5 and 21.9 to minimize their risk of disease [44] because more than three-quarters of the cases of type 2 diabetes are attributable to BMI just exceeding $21 \mathrm{~kg} / \mathrm{m}^{2}$ [45].

\subsection{Study Strengths and Limitations}

This study has several strengths. First, high-quality data were obtained from a large, representative population cohort with a high response rate. Second, the data contain several age groups, which enabled us to avoid the overgeneralization of adolescent trends over multiple age groups. Third, the survey items and data collection methods were consistent across the study years; and the timing of the studies, sampling, and data collection methods were identical throughout the study period. Fourth, given the mandatory nature of the Icelandic school system for the 14- and 15-year-olds and the high proportion of the 16- to 20-year-olds who continue their education into high-schools, in spite of the fact that schooling is not mandatory, the data collected for these age groups still encompass $70-80 \%$ of the total population in this age group. Therefore, it can be assumed that the few missing data (either from absences or improperly completed surveys) were likely randomly distributed and do not reflect a selected sub-category of the study population. Finally, this study not only identifies overweight and obesity prevalence rates but also the relative changes in pattern in underweight and normal weight and within each weight category. To date, most studies have only looked at the prevalence rates of overweight and obesity but have not focused on changes over time in the distribution of BMI, nor in the mean BMI within weight categories.

There are two limitations worth noting. First, although BMI is generally accepted as a valid indicator of body composition for purposes of population-level assessment [1,24], using BMI as an index of adiposity poses some challenges. The limitations of using BMI include inaccurate weight classifications due to high muscle-to-fat ratios, variability in the timing and tempo of adolescent 
growth spurt, and sexual maturation, all of which are potentially confounding factors [46]. Second, the use of self-reported height and weight data can be problematic, as it is known that some adolescents may tend to over-report their height, and underreport their weight; this is likely to be the case among the overweight and obese [47]. Thus, BMI values based on self-reported height and weight can under-estimate the true prevalence of overweight and obesity. However, other studies have found high correlations between measured and self-reported estimates of adolescent height and weight [17], indicating that self-reported overweight and obesity prevalence rates may be valid. For example, a Norwegian study showed that the sensitivity and specificity of self-reported BMI for identifying overweight among school children were $83 \%$ and $100 \%$, respectively [17]. Finally, unless the pattern of misreporting has changed between the survey years, the possible bias from self-reports should be the same over the measurement points and therefore not affect the overall findings.

\subsection{Implications for Practice and Future Research}

The causes of the observed increase in overweight and obesity prevalence are likely influenced by the interaction of several elements, including genetic, behavioral, psychological, and environmental factors. Changes in environmental attributes seem to be the most critical category responsible for the increase in overweight and obesity, since it would be difficult to argue for such a rapid change in genetic composition. Increases in energy consumption, decreasing energy expenditure, or a combination of both can result in energy imbalances and thus lead to increases in weight [48]. Recent social and environmental trends are only likely to affect weight levels negatively. These include a shift toward diets characterized by increased intakes of high-energy density foods, in tandem with trends toward decreased physical activity due to the increasingly sedentary nature of daily life $[1,48]$.

Our results, combined with evidence about the health consequences of obesity in adolescence and adulthood, indicate that immediate actions are needed to reverse the observed trend. For adolescents, particularly among boys, the overall BMI distribution has shifted upward, suggesting that few Icelandic adolescents and young adults are immune to the environmental influences that contribute to significant weight gain. Consequently, intervention approaches need to be broad but populationspecific, focusing on ecological factors and changes in the social and environmental determinants of youth lifestyles. Measures should be directed at settings in the society where conditions for healthy dietary habits and physical activity can be created in order to make adoption of healthy lifestyle both feasible and relatively easy. Further, although preventive efforts are more effective than treating established obesity [15], our findings suggest that community-wide preventive efforts also need to be matched by effective treatments for weight loss and weight maintenance.

\section{Conclusions}

The percentage of 14- to 20-year-old Icelandic adolescents and young adults who are overweight increased from 1992 to 2007, except among 14- and 20-year-old girls. The prevalence of obesity increased among boys in all ages, except for 16-year-olds, and among 15- and 20-year-old girls. For the adolescents, particularly among the boys, the BMI distribution has shifted upward, indicating that few Icelandic adolescents and young adults are immune to the ecological factors that appear to account 
for the observed increase in weight gain that is now being seen globally. Our findings highlight the seriousness of the increasing threat posed by the obesity problem in Iceland.

\section{Acknowledgements}

The data on which this study is based were collected by the Icelandic Centre for Social Research and Analysis, which is supported by grants from the Icelandic Alcohol and Drug Prevention Committee; the Icelandic Red Cross; the Ministry of Education, Science, and Culture; the City of Reykjavik; and the Sports and Recreational Committee of Reykjavik.

\section{References}

1. World Health Organization. Obesity: preventing and managing the global epidemic. Report of a WHO consultation. World Health Organ. Tech. Rep. Ser. 2000, 894, 1-253.

2. Diet, Nutrition and the Prevention of Chronic Disease. Joint WHO/FAO Expert Consultation; World Health Organization: Geneva, Switzerland, 2003; pp. 1-143.

3. The Challenge of Obesity in the WHO European Region and the Strategies for Response: Summary; Branca, F., Nikogosian, H., Lobstein, T., Eds.; World Health Organization Regional Office for Europe: Copenhagen, Denmark, 2007.

4. Dietz, W.H. Critical periods in childhood for the development of obesity. Am. J. Clin. Nutr. 1994, 59, 955-959.

5. Kimm, S.Y.; Barton, B.A.; Obarzanek, E.; McMahon, R.P.; Kronsberg, S.S.; Waclawiw, M.A.; Morrison, J.A.; Schreiber, G.B.; Sabry, Z.I.; Daniels, S.R. Obesity development during adolescence in a biracial cohort: the NHLBI Growth and Health Study. Pediatrics 2002, 110, e54.

6. Guo, S.S.; Chumlea, W.C. Tracking of body mass index in children in relation to overweight in adulthood. Am. J. Clin. Nutr. 1999, 70, 145S-148S.

7. Must, A.; Strauss, R.S. Risks and consequences of childhood and adolescent obesity. Int. J. Obes. Relat. Metab. Disord. 1999, 23, S2-11.

8. Bjorge, T.; Engeland, A.; Tverdal, A.; Smith, G.D. Body mass index in adolescence in relation to cause-specific mortality: a follow-up of 230,000 Norwegian adolescents. Am. J. Epidemiol. 2008, 168, 30-37.

9. Must, A.; Jacques, P.F.; Dallal, G.E.; Bajema, C.J.; Dietz, W.H. Long-term morbidity and mortality of overweight adolescents. A follow-up of the Harvard Growth Study of 1922 to 1935. N. Engl. J. Med. 1992, 327, 1350-1355.

10. Molnar, D.; Livingstone, B. Physical activity in relation to overweight and obesity in children and adolescents. Eur. J. Pediatr. 2000, 159, S45-S55.

11. Hill, A.J.; Lissau, I. Psychosocial factors. In Child and Adolescent Obesity: Causes, Consequences, Prevention, and Management; Burniat, W., Cole, T., Lissau, I., Poskitt, E., Eds.; Cambridge University Press: New York, NY, USA, 2002; pp. 109-127.

12. Sigfúsdóttir, I.D.; Kristjánsson, A.L.; Allegrante, J.P. Health behaviour and academic achievement in Icelandic school children. Health Educ. Res. 2007, 22, 70-80. 
13. Olafsson, M.; Olafsson, K.; Magnusson, K.M.; Eggertsdottir, R. The weight of school children and its relationship to performance in studies and well-being. Laeknabladid 2003, 89, 767-775. (In Icelandic)

14. Wang, Y.; Lobstein, T. Worldwide trends in childhood overweight and obesity. Int. J. Pediatr. Obes. 2006, 1, 11-25.

15. Lobstein, T.; Baur, L.; Uauy, R. Obesity in children and young people: a crisis in public health. Obes. Rev. 2004, 5, 4-104.

16. Werner, B.; Bodin, L. Obesity in Swedish schoolchildren is increasing in both prevalence and severity. J. Adolesc. Health 2007, 41, 536-543.

17. Andersen, L.F.; Lillegaard, I.T.; Overby, N.; Lytle, L.; Klepp, K.I.; Johansson, L. Overweight and obesity among Norwegian schoolchildren: changes from 1993 to 2000. Scand. J. Public. Health 2005, 33, 99-106.

18. Matthiessen, J.; Velsing Groth, M.; Fagt, S.; Biltoft-Jensen, A.; Stockmarr, A.; Andersen, J.S.; Trolle, E. Prevalence and trends in overweight and obesity among children and adolescents in Denmark. Scand. J. Public. Health 2008, 36, 153-160.

19. Kautiainen, S. Trends in adolescent overweight and obesity in the Nordic countries Scandinavian J. Food Nutr. 2005, 49, 4-14.

20. Olds, T.S.; Tomkinson, G.R.; Ferrar, K.E.; Maher, C.A. Trends in the prevalence of childhood overweight and obesity in Australia between 1985 and 2008. Int. J. Obes. (Lond) 2010, 34, 57-66.

21. Ogden, C.L.; Carroll, M.D.; Flegal, K.M. High body mass index for age among US children and adolescents, 2003-2006. JAMA 2008, 299, 2401-2405.

22. Peneau, S.; Salanave, B.; Maillard-Teyssier, L.; Rolland-Cachera, M.F.; Vergnaud, A.C.; Mejean, C.; Czernichow, S.; Vol, S.; Tichet, J.; Castetbon, K.; Hercberg, S. Prevalence of overweight in 6to 15-year-old children in central/western France from 1996 to 2006: trends toward stabilization. Int. J. Obes. (Lond) 2009, 33, 401-407.

23. Jolliffe, D. Extent of overweight among US children and adolescents from 1971 to 2000. Int. J. Obes. Relat. Metab. Disord. 2004, 28, 4-9.

24. Guillaume, M.; Lissau, I. Epidemiology. In Child and Adolescent Obesity. Causes and Consequences, Prevention and Management; Burniat, W., Cole, T., Lissau, I., Poskitt, E., Eds.; Cambridge University Press: New York, NY, USA, 2002; pp. 28-49.

25. Pálsdóttir, H.; Kristjánsson, Á.L.; Sigfúsdóttir, I.D.; Baldursdóttir, B.; Sigfússon, J. Ungt fólk utan skóla 2009. Félagsleg staða 16-20 ára ungmenna á Íslandi sem ekki stunda nám við framhaldsskóal árið 2009. Young people not attending schools in 2009. The social position of 16-20-year old Icelandic adolescents who do not attend high-schools 2009. Rannsóknir og greining: Reykjavík, Iceland, 2010; pp. 1-178. (In Icelandic)

26. Youth in Iceland Data Collection Protocol; Icelandic Centre for Social Research and Analysis: Reykjavík, Iceland, 2007.

27. Cole, T.J.; Bellizzi, M.C.; Flegal, K.M.; Dietz, W.H. Establishing a standard definition for child overweight and obesity worldwide: international survey. BMJ 2000, 320, 1240-1243.

28. Cole, T.J.; Flegal, K.M.; Nicholls, D.; Jackson, A.A. Body mass index cut offs to define thinness in children and adolescents: international survey. BMJ 2007, 335, 194. 
29. Daviglus, M.L.; Liu, K.; Yan, L.L.; Pirzada, A.; Manheim, L.; Manning, W.; Garside, D.B.; Wang, R.; Dyer, A.R.; Greenland, P.; Stamler, J. Relation of body mass index in young adulthood and middle age to Medicare expenditures in older age. JAMA 2004, 292, 2743-2749.

30. Stewart, S.T.; Cutler, D.M.; Rosen, A.B. Forecasting the effects of obesity and smoking on U.S. life expectancy. N. Engl. J. Med. 2009, 361, 2252-2260.

31. Valdimarsdóttir, M.; Jónsson, S.H.; Porgeirsdóttir, H.; Gísladóttir, E.; Guðlaugsson J.Ó.; Pórlindsson, P. Likamspyngd og holdarfar fullorðinna Íslendinga frá 1990 til 2007 [Body weight and physique of Icelandic adults from 1990 to 2007.]; Lýðheilsustöð: Reykjavík, Iceland, September, 2009. (In Icelandic)

32. EU Platform on Diet, Physical Activity and Health; International Obesity Task Force and the European Association for the Study of Obesity: Brussels, Belgium, March 15, 2005.

33. Whitaker, R.C.; Wright, J.A.; Pepe, M.S.; Seidel, K.D.; Dietz, W.H. Predicting obesity in young adulthood from childhood and parental obesity. N. Engl. J. Med. 1997, 337, 869-873.

34. Sebire, N.J.; Jolly, M.; Harris, J.P.; Wadsworth, J.; Joffe, M.; Beard, R.W.; Regan, L.; Robinson, S. Maternal obesity and pregnancy outcome: a study of 287,213 pregnancies in London. Int. J. Obes. Relat. Metab. Disord. 2001, 25, 1175-1182.

35. Johannsson, E.; Arngrimsson, S.A.; Thorsdottir, I.; Sveinsson, T. Tracking of overweight from early childhood to adolescence in cohorts born 1988 and 1994: overweight in a high birth weight population. Int. J. Obes. (Lond) 2006, 30, 1265-1271.

36. Manneldisráð Íslands. Hvað borða Íslendingar. Könnun á matarcæi Íslendinga 2002. Helstu niðurstöður. The diet of Icelanders. Dietary survey of the Icelandic Nutrition Council 2002. The main findings. Manneldisráo Íslands: Reykjavík, Iceland, 2002; pp. 1-103. (In Icelandic)

37. Janssen, I.; Katzmarzyk, P.T.; Boyce, W.F.; Vereecken, C.; Mulvihill, C.; Roberts, C.; Currie, C.; Pickett, W. Comparison of overweight and obesity prevalence in school-aged youth from 34 countries and their relationships with physical activity and dietary patterns. Obes. Rev. 2005, 6, 123-132.

38. Croezen, S.; Visscher, T.L.; Ter Bogt, N.C.; Veling, M.L.; Haveman-Nies, A. Skipping breakfast, alcohol consumption and physical inactivity as risk factors for overweight and obesity in adolescents: results of the E-MOVO project. Eur. J. Clin. Nutr. 2009, 63, 405-412.

39. Eithsdottir, S.T.; Kristjánsson, A.L.; Sigfúsdóttir, I.D.; Allegrante, J.P. Trends in physical activity and participation in sports clubs among Icelandic adolescents. Eur. J. Public. Health 2008, 18, 289-293.

40. Willett, W.C.; Dietz, W.H.; Colditz, G.A. Guidelines for healthy weight. N. Engl. J. Med. 1999, 341, 427-434.

41. Kenchaiah, S.; Evans, J.C.; Levy, D.; Wilson, P.W.; Benjamin, E.J.; Larson, M.G.; Kannel, W.B.; Vasan, R.S. Obesity and the risk of heart failure. N. Engl. J. Med. 2002, 347, 305-313.

42. Kurth, T.; Gaziano, J.M.; Berger, K.; Kase, C.S.; Rexrode, K.M.; Cook, N.R.; Buring, J.E.; Manson, J.E. Body mass index and the risk of stroke in men. Arch. Intern. Med. 2002, 162, 2557-2562.

43. Nathan, B.M.; Moran, A. Metabolic complications of obesity in childhood and adolescence: more than just diabetes. Curr. Opin. Endocrinol. Diabetes Obes. 2008, 15, 21-29. 
44. Field, A.E.; Coakley, E.H.; Must, A.; Spadano, J.L.; Laird, N.; Dietz, W.H.; Rimm, E.; Colditz, G. A. Impact of overweight on the risk of developing common chronic diseases during a 10 -year period. Arch. Intern. Med. 2001, 161, 1581-1586.

45. James, W.P.T.; Jackson-Leach, R.; Mhurchu, C.N.; Kalamara, E.; Shayeghi, M.; Gigby, N.J.; Nishida, C.; Rodgers, A. Overweight and obesity (high body mass index). In Comparative Quantification of Health Risks: Global and Regional Burden of Disease Attribution to Selected Major Risk Factors; Ezzati, M., Lopez, A.D., Rodgers, A., Murray, C.J.L., Eds.; World Health Organization: Geneva, Switzerland, 2004; pp. 497-596.

46. Malina, R.M.; Katzmarzyk, P.T. Validity of the body mass index as an indicator of the risk and presence of overweight in adolescents. Am. J. Clin. Nutr. 1999, 70, 131S-136S.

47. Fortenberry, J.D. Reliability of adolescents' reports of height and weight. J. Adolesc. Health. 1992, 13, 114-117.

48. Stein, C.J.; Colditz, G.A. The epidemic of obesity. J. Clin. Endocrinol. Metab. 2004, 89, $2522-2525$.

(C) 2010 by the authors; licensee MDPI, Basel, Switzerland. This article is an open-access article distributed under the terms and conditions of the Creative Commons Attribution license (http://creativecommons.org/licenses/by/3.0/). 